\title{
A comparison of type II operating characteristics derived from confidence ratings and from latencies ${ }^{1}$
}

\author{
LEONARD KATZ ${ }^{2}$ \\ UNIVERSITY OF CONNECTICUT
}

On each of a series of trials, $S$ judged two stimuli to be the same or different $S$ then made a confidence rating of his judgment. The difficulty of discrimination and feedback of response information (latency and correctness) were manipulated. Feedback had no effect. Latencies conditional on confidence rating decreased with increasing confidence and remained relatively constant with changes in discrimination difficulty. Type II operating characteristics were derived from latencies and from confidence ratings; both appropriately reflected differences in task difficulty.

The present experiment studies the relationship between confidence rating and latency in a matching task with two levels of difficulty. Investigators working in perception and short-term memory have made use of the confidence rating technique derived from signal detectability theory by Pollack \& Decker (1958) in order to obtain Type II (i.e., response conditional) operating characteristics (OCs). The construction of OCs is usually more efficient when $S$ is allowed to choose from several subjective criteria of decision certainty within an experimental session than when $\mathbf{S}$ adopts differential certainty criteria that are manipulated by $\mathrm{E}$ between sessions.

A method for producing OCs from latency data has been presented for a choice reaction-time (RT) situation by Carterette et al (1965). The keystone of the Carterette et al technique is the trade-off between speed and accuracy that is usually found for choice RT (cf. Fitts, 1966). In the choice RT situation, the stimulus remains on at least until $S$ responds. Thus, $S$ is free to fix the criterion necessary to effect a given response and can sample the stimulus (whatever the signal-to-noise ratio) until one of his criteria is satisfied. The RT for error responses should be smaller than the RT for correct responses.

In contrast, the $\mathrm{E}$ terminates the stimulus before $\mathrm{S}$ responds, in many perception experiments (e.g., the present experiment). The proximal representation of the stimulus decays rapidly after stimulus presentation (or it becomes degraded, becomes less available, etc.).
Similarly, in experiments where $S$ is required to recognize or recall, the appropriate memory may decay (become degraded, less available, etc.) as a function of time since stimulus presentation. In both kinds of experiments, $S$ must attempt to respond to a stimulus that has a decreasing signal-to-noise ratio and, therefore, a decreasing likelihood ratio. Models based on this notion must predict mean RT on error responses to be larger than RT on correct responses (at least after some relatively brief initial period). Also, RT should decrease as confidence increases. $^{3}$

A relatively simple stimulus matching task was chosen. The basic nature of the decision, "This stimulus is different from that," has been indicated by Luce (1963) and, more recently, by Posner and Mitchell (1967). Luce has placed the matching experiment in logical relation to the variety of experimental paradigms used for the study of choice and decision behavior. Posner and Mitchell discussed the connection between the matching experiment and the recognition experiment and indicated the importance of matching behavior in memory processes. Wickelgren and Norman (e.g., 1966), among others, have utilized assumptions about matching behavior in their work on short-term recognition memory.

In the present study, $S$ observed, on each of a series of trials, two stimuli that were presented simultaneously for a brief duration (less than $.5 \mathrm{sec}$ ). $\mathrm{S}$ responded with a "same" or "different" judgment followed by a confidence rating of his decision. The dependent variables of interest were the latency of the judgment and the confidence rating of the judgment. Operating characteristics were computed from latencies by a method analogous to that used for confidence ratings. First, each S's latencies were partitioned into four categories, "fast," "moderately fast," "moderately slow," and "slow." Then the proportions of correct-response latencies in each category and the proportions of incorrect-response latencies in each category were computed. Lastly, cumulative proportions were computed, giving a 3-point $O C$ in which each point was determined by the cumulative proportions of correct- and incorrect-response latencies in a given category. Each $O C$ derived from latencies was compared with the $\mathrm{OC}$ derived from confidence ratings based on the same set of trials.

The level of task difficulty and the immediate feedback of response information (latency and correctness) were manipulated in order to test the invariance of the correspondence between a given confidence rating and its mean latency. With regard to task difficulty, the comparison between rating and latency OCs is facilitated if their equivalence relation does not change with changes in sensitivity, i.e., with changes in $d_{r}$ or a similar index. With regard to the manipulation of immediate feedback, it was felt that $S$ would become less confident when deprived of feedback and, therefore, would alter his pattern of ratings even though his sensitivity should remain equal to his sensitivity in an immediate feedback condition of equal difficulty. Again, interpretation of the data would be simplified if the criterion-latency relation remained constant as the relative use of each confidence rating changed.

\section{METHOD}

\section{Apparatus}

A Digital Equipment Corporation PDP.5 computer was used in conjunction with a cathode ray tube (CRT) to produce and display one of four stimulus pattems on each trial. The four stimuli were (1) two solid horizontal lines, each 2 in. in length, which formed two sides of an imaginary square, (2) two lines similar to the first pair but with a gap in the center of each, (3) two lines similar to the first pair but with a gap in the upper line, the lower line being solid, and (4) two lines similar to the first pair but with a gap in the lower line, the upper line being solid. Each of the four was presented to $S$ in a demonstration series during which he was told to respond "same" to Stimuli 1 and 2 and "different" to Stimuli 3 and 4 . The stimulus intensity was set separately for each $\mathbf{S}$ in order to produce error rates that were neither extremely high nor low. From one to three intensity adjustments were made during a practice series of trials. Because the computer cycled only once through a 96 by 85 dot matrix for each stimulus 
display (total time equaled $25 \mathrm{msec}$ ), the stimulus intensity alone determined the strength of the glow on the CRT and, therefore, determined the stimulus duration. Each dot was intensified for 5 microsec and decayed to $10 \%$ of its initial value in $.85 \mathrm{msec}$. All the intensities used produced displays that were, subjectively, dim. Intensity differences between $\mathrm{Ss}$ were small.

The stimuli were presented in 12 blocks of 60 each, the order of the four stimuli being random under the constraint that each appear 15 times in each block. Preceding each block were four dummy trials that were not analyzed.

The size of the stimulus gap (difficulty level) and feedback of information (feedback) were varied factorially between blocks. For a given block, the size of the gap was either subjectively small (approximately $1 / 32$ in.), termed Gap 1 (G1), or large (approximately 1/16 in.), Gap 2 (G2). For a given block, $S$ either received immediate feedback (IF) of his latency and correctness following each response or he received no immediate feedback (NIF). Under IF, S's reaction time in 10ths of a second and the stimulus type (e.g., $S$ or D) were displayed on the CRT at the end of each trial. At the end of the block, S observed a Teletype reproduce the same trial-by-trial information together with S's actual responses. Under NIF, the CRT was blank between stimulus presentations, and $S$ observed only the Teletype output at the end of a block.

$S$ received four blocks of practice trials, in the order G2-1F, G2-NIF, G1-1F, G1-NIF, followed by two repetitions of the same sequence of blocks for the experimental run. The sequence of stimuli was randomized differently for each $S$ and within each of the 12 blocks. Thus, each $S$ received 30 practice trials and 60 experimental trials in each of the eight combinations of difficulty level, feedback, and stimulus type.

\section{Procedure}

The $S$ sat in a darkened cubicle approximately $3 \mathrm{ft}$ from the CRT. A switch matrix lay under his dominant hand; it contained a start button, a "same" button, a "different" button, and three confidence rating buttons. The position of the "same" and "different" buttons was counterbalanced across Ss. S initiated a trial by pressing the start button and $.5 \mathrm{sec}$ later a stimulus was displayed. Reaction time was measured from stimulus onset until $S$ pressed either the "same" or "different" button. $S$ then made a confidence rating of his choice of "same" or "different" by pressing one of the three confidence rating buttons, termed 1 -least

Table 1

Mean Latencies (in Sec) and Frequencies (in Parentheses) for Correct- and Incorrect-Responses in Each Difficulty Level and Stimulus Type Combination

\begin{tabular}{llccccr}
\hline & \multicolumn{7}{c}{ Subject } \\
\cline { 2 - 7 } \multicolumn{1}{c}{ Condition } & DW & BL & DK & RW & Mean \\
\hline Gap 1 & & & & & \\
$\quad$ Correct, Same & $1.11(73)$ & $.83(81)$ & $1.15(78)$ & $1.09(89)$ & 1.04 \\
$\quad$ Correct, Different & $1.15(81)$ & $.93(51)$ & $1.21(66)$ & $1.33(81)$ & 1.15 \\
$\quad$ Incorrect, Same & $1.30(47)$ & $1.06(39)$ & $1.09(42)$ & $1.63(31)$ & 1.27 \\
Incorrect, Different & $1.17(39)$ & $.77(69)$ & $1.18(54)$ & $1.59(39)$ & 1.18 \\
Gap 2 & & & & & \\
$\quad$ Correct, Same & $.93(97)$ & $.68(110)$ & $.93(104)$ & $.90(96)$ & .86 \\
$\quad$ Correct, Different & $.86(111)$ & $.85(11)$ & $.88(97)$ & $1.00(112)$ & .89 \\
$\quad$ Incorrect, Same & $1.28(23)$ & $1.14(10)$ & $1.06(16)$ & $1.38(24)$ & 1.21 \\
Incorrect, Different & $1.14(9)$ & $1.20(9)$ & $1.09(23)$ & $1.33(8)$ & 1.19 \\
\hline
\end{tabular}

confident, 2-moderately contident, and $3-$ most confident. $S$ was encouraged to make his confidence rating quickly. Under Condition IF, S's latency, together with an appropriate " $S$ " or " $D$ " describing the stimulus, was displayed when a confidence rating button was pressed. Under NIF, the screen remained blank. A block of trials took 12 to $14 \mathrm{~min}$ to complete. S left the cubicle for $5 \mathrm{~min}$ after each block to observe the Teletype output; he took longer rests of about $15 \mathrm{~min}$ each after the practice series and after the eighth block.

\section{Subjects}

Ss were four volunteers who were paid $\$ 12$ each for participating. One $\mathrm{S}$ was a Psychology Department staff member and the remaining Ss were students. All were naive with respect to the main purpose of the experiment.

\section{RESULTS AND DISCUSSION}

\section{Mean Latency and Condition}

The analyses of main interest required partitioning data into correct and incorrect responses. Because some Ss made few correct responses in some conditions (e.g., the "least-confident" category under the easy discrimination) and for the reason discussed next, data were first aggregated over feedback condition to ensure sufficient data. Reports from Ss suggested that the feedback manipulation had no important effect on either ratings or latencies; inspection of the data confirmed this. Two factors may have contributed to attenuating any possible feedback effect: (1) Ss received alternating blocks of IF and NIF and (2) they received feedback via the Teletype after every block and, therefore, may have had sufficient feedback to stabilize their use of the three confidence ratings and the related latencies.

Table 1 presents the mean latencies for each $S$ in each combination of difficulty level (Gap 1, difficult; Gap 2, easy) and stimulus type (same, different) for correct and incorrect responses. Each mean is based on a variable number of observations; frequencies are in parentheses. Inspection of the data suggests that latencies on incorrect responses are longer than latencies on correct responses. - Incorrect-response latencies do not appear to be affected by difficulty level. However, correct-response latencies appear to decrease with decreasing difficulty.

Mean Latency, Confidence Ratings, and Percent Correct

The data were analyzed to assess the effects on mean latency of difficulty level, stimulus type, correctness, and confidence rating. A 2 by 2 by 2 by 3 repeated-measurements analysis of variance was performed; each mean latency for each $S$ was based on a minimum of four responses.

The major results are presented in Fig. 1. The analysis of variance suggested that the effect of confidence rating was strong; as confidence increased, mean latency decreased, $F(2,6)=20.12, p<.01$. Neither difficulty level nor any of its interactions approached significance and, in fact, the mean square for difficulty level was small.

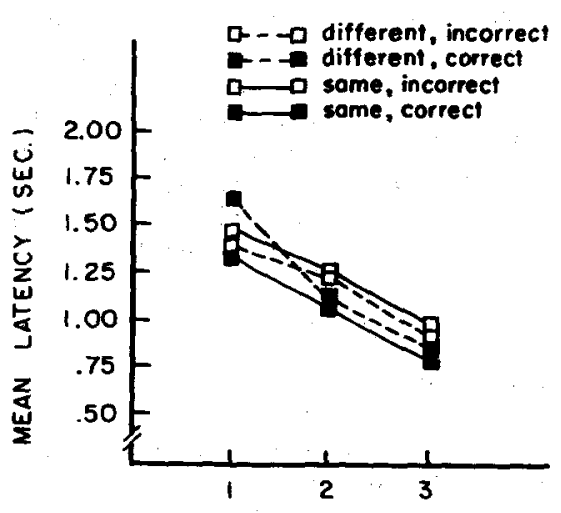

CONFIDENCE RATING

Fig. 1. Mean latency as a function of confidence rating for each of four combinations of stimulus type and correctness. 
Table 2

Mean Latencies (in Sec), Per Cent Correct, and Number of Observations (in Parentheses) as a Function of Confidence Rating and Difficulty Level

\begin{tabular}{|c|c|c|c|c|c|c|c|c|c|c|c|c|c|}
\hline \multirow{2}{*}{\multicolumn{2}{|c|}{$\begin{array}{c}\text { Confi- } \\
\text { Condi- dence } \\
\text { tion Rating }\end{array}$}} & \multicolumn{12}{|c|}{ Subject } \\
\hline & & \multicolumn{3}{|c|}{ DW } & \multicolumn{3}{|c|}{ BL } & \multicolumn{2}{|r|}{ DK } & \multicolumn{2}{|r|}{ RW } & \multicolumn{2}{|c|}{$\begin{array}{c}\text { Mean } \\
\text { Latency }\end{array}$} \\
\hline Gap 1 & $\begin{array}{l}1 \\
2 \\
3\end{array}$ & $\begin{array}{r}1.54 \\
1.31 \\
.94\end{array}$ & $\begin{array}{l}53 \\
66 \\
67\end{array}$ & $\begin{array}{l}(45) \\
(104) \\
(91)\end{array}$ & $\begin{array}{r}1.12 \\
.94 \\
.71\end{array}$ & $\begin{array}{l}41 \\
57 \\
63\end{array}$ & $\begin{array}{l}(69) \\
(75) \\
(96)\end{array}$ & $\begin{array}{l}1.55 \\
1.20 \\
1.02\end{array}$ & $\begin{array}{ll}58 & (50) \\
60 & (45) \\
59 & (145)\end{array}$ & $\begin{array}{r}1.79 \\
1.22 \\
.91\end{array}$ & $\begin{array}{l}60 \\
71 \\
85\end{array}$ & $\begin{array}{l}(95) \\
(66) \\
(79)\end{array}$ & $\begin{array}{r}1.50 \\
1.17 \\
.90\end{array}$ \\
\hline Gap 2 & $\begin{array}{l}1 \\
2 \\
3\end{array}$ & $\begin{array}{r}1.33 \\
1.17 \\
.80 \\
\end{array}$ & $\begin{array}{l}43 \\
72 \\
96 \\
\end{array}$ & $\begin{array}{l}(30) \\
(49) \\
(161)\end{array}$ & $\begin{array}{r}1.06 \\
1.02 \\
.99 \\
\end{array}$ & $\begin{array}{l}76 \\
92 \\
96\end{array}$ & $\begin{array}{l}(37) \\
(49) \\
(154)\end{array}$ & $\begin{array}{r}1.40 \\
1.24 \\
.86 \\
\end{array}$ & $\begin{array}{l}48 \\
82 \\
92 \\
90\end{array}$ & $\begin{array}{r}1.60 \\
1.31 \\
.88\end{array}$ & $\begin{array}{l}66 \\
79 \\
95\end{array}$ & $\begin{array}{l}(48) \\
(42) \\
(150)\end{array}$ & $\begin{array}{r}1.35 \\
1.18 \\
.88\end{array}$ \\
\hline
\end{tabular}

Thus, it appears that the mean latency associated with each confidence rating remained stable as task difficulty changed. Neither stimulus type nor correctness were significant. However, the Stimulus Type by Correctness interaction was significant, $F(1,3)=13.88, p<.05$. The effect appears to be due to the smaller mean latency of the "same" stimulus correct-response condition relative to the other three conditions. The magnitude of the effect is small compared to the effect of confidence rating.

The relative constancy of the latency-confidence rating relation can be evaluated more closely with the data in Table 2. Table 2 presents the mean latencies, percent correct, and number of observations for each $S$ as a function of confidence rating and difficulty level. Except for SBL, each S's two mean latencies for a given confidence rating tend to be closer to each other than to the latencies for other confidence ratings. The correspondences ate better for the "moderately confident" and "most confident" ratings. S BL's data show relatively small changes as a function of rating and difficulty. The average across Ss and the results of the analysis of variance mentioned above, suggest that considerably smaller variability occurs between latencies with the same confidence rating from different difficulty levels than between latencies with different confidence ratings at a given difficulty level.

Pollack and Decker (1958) found that the average percent correct message receptions conditionalized on confidence rating did not change substantially with differences in the signal-to-noise ratio $(\mathrm{S} / \mathrm{N})$ of the messages. Their result is analagous to the latency-confidence rating constancy found in the present experiment. However, percent correct in the present study does not display the constancy found by Pollack and Decker. The data of Table 2 suggest that Ss shifted their decision criteria with changes in task difficulty; they adopted criteria for the easy task that were more conservative than criteria in the difficult task. Thus, a given confidence rating had a subjectively different meaning in each difficulty level.

It should be noted that the Pollack and Decker (1958) result held only for message sets of different $S / N$ ratios; when their data were partitioned into subsets of messages of different difficulties (without regard to $\mathrm{S} / \mathrm{N}$ ) they found that the proportion of correct responses given a "most confident" rating was highest for the least difficult subset. This latter result is similar to the result obtained in the present study with percent correct as the measure.

These results suggest that (1) the latency of responses increases with decreasing confidence rating, (2) S may shift the subjective confidence criterion (corresponding to a given confidence rating) with changes in task difficulty, and (3) the latency associated with a given confidence rating remains invariant with a shift in the subjective criterion for that confidence rating.

\section{Operating Characteristics}

Operating characteristics for confidence ratings were computed separately for each $S$ by the method of Pollack and Decker (1958). Frequencies of each rating for each

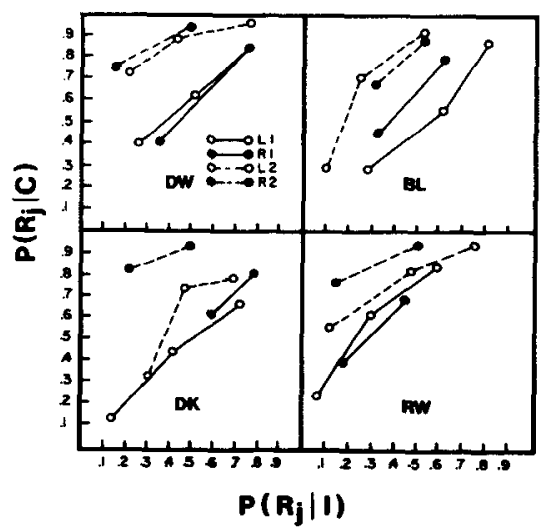

Fig. 2. Operating characteristics based on latencies and on confidence ratings for each of four Ss. The OCs for the difficult task (Gap 1) are labeled $L 1$ and $R 1$, respectively, for latencies and ratings. The OCs for the easy task (Gap 2) are labeled $L 2$ and $R 2$.
$S$ were aggregated across stimulus type and feedback to produce two 3 by 2 matrices (Confidence Rating by Correctness), one matrix for each difficulty level. There was a total of 240 responses in each matrix for each S. For each level of correctness (correct, C, and incorrect, I) in each matrix, the proportion of responses that was given confidence rating $J$ or higher was computed $(j=1,2,3)$, giving the proportions $P\left(R_{j} \mid C\right)$ and $P\left(R_{j} \mid I\right)$. This produced 2-point OCs in which $P\left(R_{j} \mid C\right)$ is the ordinate and $P\left(R_{j} \mid I\right)$ the abscissa. Figure 2 presents the rating OCs for Gap 1 (R1) and Gap 2 (R2) for each of four Ss.

The latency OCs corresponding to each of the rating OCs were computed separately for each $S$ by a method analogous to the confidence rating technique. First, four categories of latency were determined from 1-slowest to 4-fastest. The latency criteria that corresponded to the category bounds were chosen separately for each $S$ in order to ensure sufficient data in all categories. For example, the widest distribution of latencies was given by S RW whose categories, 4 through 1 , respectively, were partitioned as follows: $0-8 \mathrm{sec}, .9-1.1 \mathrm{sec}$, $1.1-1.5 \mathrm{sec}, 1.6 \mathrm{sec}$ and longer. The narrowest distribution was given by $\mathrm{S} B L$ whose latencies were partitioned as follows: $\quad 0-.6 \mathrm{sec}, \quad .7-.8 \mathrm{sec}, \quad .9-1.1 \mathrm{sec}$, $1.2 \mathrm{sec}$ and longer. No $\mathrm{S}$ produced more than two latencies below $.5 \mathrm{sec}$.

Frequencies of each category for each $\mathrm{S}$ were aggregated across stimulus type, feedback, and confidence rating to produce two 4 by 2 matrices of Latency Category by Correctness, one matrix for each difficulty level. For each level of correctness in each matrix, the proportion of responses in Category $j$ or higher was computed, giving the proportions $P\left(R_{j} \mid C\right)$ and $P\left(R_{j} \mid I\right)$, where $R_{j}$ now refers to the $j$ th latency response category and has the range $\mathrm{j}=1,2,3,4$. This produced the 3-point latency OCs presented in Fig. 2 for Gap 1 (L1) and Gap 2 (L2) for each of four Ss.

Comparisons between Gap 1 and Gap 2 within latency and within ratings indicates separation in the appropriate direction; R2 is farther from the positive diagonal than $R 1$, and $L 2$ is generally farther from the diagonal than $\mathrm{L} 1$. However, the relation between latency and rating $\mathrm{OCs}$ is not systematic; each $S$ displays a different pattern of correspondence. These differences do not appear to be related to the different latency category bounds that were chosen. Inspection of Fig. 2, together with the results of the analysis of variance, suggests that latency and rating OCs based on group data would show a reasonable correspondence. Thus, the use of latency 
data, in lieu of confidence ratings, to produce Type II OCs appears to be worthy of further consideration.

\section{REFERENCES}

BINDRA, D., WILLIAMS, J. A., \& WISE, J. S. Judgments of sameness and difference: Experiments on decision time. Science, 1965, 150, 1625-1627.

CARTERETTE, E. C., FRIEDMAN, M. P., \& COSMIDES, R. Reaction-time distributions in the detection of weak signals in noise. Journal of the Acoustical Society of America, 1965, $38,531-542$.

FITTS, P. M. Cognitive aspects of information processing: III. Set for speed versus accuracy. Journal of Experimental Psychology, 1966, 71, 849-857.
LUCE, R. P. Discrimination. In R. P. Luce, X. X. Bush, and E. Galanter (Eds.), Handbook of mathematical psychology. New York: Wiley, 1963.

NORMAN, D. A., \& WICKELGREN, W. A. Strength theory of decision rules and latency in retrieval from short-term memory. Joumal of Mathematical Psychology, 1969, 6, 192-208.

POLLACK, I., \& DECKER, L. R. Confidence ratings, message reception, and the receiver operating characteristics. Journal of the Acoustical Society of America, 1958, 30 286-292.

short-term recognition memory. Joumal of Mathematical Psychology, 1966, 3, 316-347.

POSNER, M. I., \& MITCHELL, R. F. Chronometric analysis of classification. Psychological Review, 1967, 74, 392-409.

WICKELGREN, W. A., \& NORMAN, D. A

Strength models and serial position in (Accepted for pubizcation October 20, 1969.) 